Address correspondence to: Charles T. Esmon, Oklahoma Medical Research Foundation, Cardiovascular Biology, Howard Hughes Medical Research Foundation, 825 N.E. 13th Street, Oklahoma City, Oklahoma 73104, USA. Phone: (405) 271-6474; Fax: (405) 271-2872; E-mail: Charles-Esmon@omrf.org.

1. Mitsumoto, H., Chad, D.A., and Pioro, E.P. 1998. Amyotrophic lateral sclerosis. F.A. Davis. Philadelphia, Pennsylvania, USA. 480 pp.

2. Garbuzova-Davis, S., et al. 2007. Ultrastructure of blood-brain barrier and blood-spinal cord barrier in SOD1 mice modeling ALS. Brain Res. 1157:126-137.

3. Zhong, Z., et al. 2008. ALS-causing SOD1 mutants generate vascular changes prior to motor neuron degeneration. Nat. Neurosci. 11:420-422.

4. Zhong, Z., et al. 2009. Activated protein C therapy slows ALS-like disease in mice by transcriptionally inhibiting SOD1 in motor neurons and microglia cells. J. Clin. Invest. 119:3437-3449.

5. Esmon, C.T., and Schwarz, H.P. 1995. An update on clinical and basic aspects of the protein $\mathrm{C}$ anticoagulant pathway. Trends Cardiovasc. Med. 5:141-148.

6. Bernard, G.R., et al. 2001. Efficacy and safety of recombinant human activated protein $\mathrm{C}$ for severe sepsis. N. Engl. J. Med. 344:699-709.

7. Scaldaferri, F.F., et al. 2007. Crucial role of the pro- tein $\mathrm{C}$ pathway in governing microvascular inflammation in inflammatory bowel disease. J. Clin. Invest. 117:1951-1960.

8. Isermann, B., et al. 2007. Activated protein C protects against diabetic nephropathy by inhibiting endothelial and podocyte apoptosis. Nat. Med. 13:1349-1358

9. Cheng, T., et al. 2003. Activated protein C blocks p53-mediated apoptosis in ischemic human brain endothelium and is neuroprotective. Nat. Med. 9:338-342.

10. Van Sluis, G.L., et al. 2009. Endogenous activated protein C limits cancer cell extravasation through sphingosine-1-phosphate receptor 1 mediated vascular endothelial barrier enhancement. Blood. 114:1968-1973.

11. Han, M.H., et al. 2008. Proteomic analysis of active multiple sclerosis lesions reveals therapeutic targets. Nature. 451:1076-1083.

12. Mizutani, A., Okajima, K., Uchiba, M., and Noguchi, T. 2000. Activated protein C reduces ischemia/ reperfusion-induced renal injury in rats by inhibiting leukocyte activation. Blood. 95:3781-3787.

13. Laszik, Z., Mitro, A., Taylor, F.B., Jr., Ferrell, G., and Esmon, C.T. 1997. Human protein C receptor is present primarily on endothelium of large blood vessels: implications for the control of the protein C pathway. Circulation. 96:3633-3640.

14. Nakanishi-Matsui, M., et al. 2000. PAR3 is a cofactor for PAR4 activation by thrombin. Nature. 404:609-613.

15. Riewald, M., Petrovan, R.J., Donner, A., and Ruf, W. 2003. Activated protein C signals through the thrombin receptor PAR1 in endothelial cells. J. Endotoxin Res. 9:317-321.

16. Bae, J.S., Yang, L., Manithody, C., and Rezaie, A.R. 2007. The ligand occupancy of endothelial protein $C$ receptor switches the protease-activated receptor 1 -dependent signaling specificity of thrombin from a permeability-enhancing to a barrier-protective response in endothelial cells. Blood. 110:3909-3916.

17. Mosnier, L.O., Zlokovic, B.V., and Griffin, J.H. 2007. The cytoprotective protein C pathway. Blood. 109:3161-3172.

18. McIntosh, D.P., Tan, X.Y., Oh, P., and Schnitzer, J.E. 2002. Targeting endothelium and its dynamic caveolae for tissue-specific transcytosis in vivo: a pathway to overcome cell barriers to drug and gene delivery. Proc. Natl. Acad. Sci. U. S. A. 99:1996-2001.

19. Apostolski, S., et al. 1991. Serum and CSF immunological findings in ALS. Acta Neurol. Scand. 83:96-98.

20. Leonardi, A., Abbruzzese, G., Arata, L., Cocito, L., and Vische, M. 1984. Cerebrospinal fluid (CSF) findings in amyotrophic lateral sclerosis. J. Neurol. 231:75-78.

21. Glass, J.D., Benatar, M., and Polak, M. 2007. Selecting promising ALS therapies in clinical trials. Neurology. 68:1545-1546.

22. James, J.A., Gross, T., Scofield, R.H., and Harley, J.B. 1995. Immunoglobulin epitope spreading and autoimmune disease after peptide immunization: Sm B/B'-derived PPPGMRPP and PPPGIRGP induce spliceosome autoimmunity. J. Exp. Med. 181:453-461.

\title{
Smad4: gatekeeper gene in head and neck squamous cell carcinoma
}

\author{
Murray Korc \\ Departments of Medicine, Pharmacology, and Toxicology, and Norris Cotton Cancer Center, Dartmouth-Hitchcock Medical Center, \\ Lebanon, New Hampshire, USA. Dartmouth Medical School, Hanover, New Hampshire, USA.
}

\begin{abstract}
Unchecked cell growth is a hallmark of cancer. During oncogenesis, cancerous cells become resistant to the TGF- $\beta$ signaling pathway that usually keeps cell growth in check. The role of a critical mediator of this pathway, Smad4, in head and neck squamous cell carcinoma (HNSCC) remains unclear. In this issue of the JCI, Bornstein and colleagues report that Smad4 expression is decreased in malignant HNSCC and, surprisingly, also in normal-appearing buccal mucosa adjacent to HNSCC (see the related article beginning on page 3408). They also show that targeted conditional deletion of Smad4 in the head and neck epithelium of mice is alone sufficient to initiate spontaneous HNSCC, in conjunction with DNA repair gene dysregulation, genetic instability, and inflammation. These findings point to a novel function for Smad4 as a guardian gene that maintains genomic stability.
\end{abstract}

The vast majority of head and neck cancers are squamous cell carcinomas (HNSCCs), all of which arise from a mucosal surface. HNSCCs can include cancers of the mouth, larynx, pharynx, tongue, lip, or nasal cavity

Conflict of interest: The author has declared that no conflict of interest exists.

Citation for this article: J. Clin. Invest. 119:3208-3212 (2009). doi:10.1172/JCI41230. but traditionally do not include cancers of the thyroid, esophagus, or skin. The malignancy is more prevalent in males and in individuals who smoke or chew tobacco and/or consume alcohol $(1,2)$. Certain viral agents, such as human papilloma virus types 16 and 18, increase the risk of developing HNSCC in the oral cavity (1, 2). In spite of considerable advances in our understanding of the molecular alterations that occur in this malignancy, the 5-year survival rate has stubbornly remained at approximately $50 \%$, due to resistance to therapy; cancer recurrence following surgical resection even when followed by chemoor radiotherapy; and the development of second, unrelated malignancies (1-3).

Key genetic alterations known to exist in HNSCC include (a) overexpression of the growth factor receptor EGFR; (b) mutations in the tumor suppressor gene $p 53$; (c) mutation or overexpression of the oncogenes $\mathrm{K}$-ras or $\mathrm{H}$-ras; (d) increased levels of the cell-cycle regulator and proto-oncogene cyclin D1, the cytokine IL- 6 , the transcription factor runt-related transcription factor 2 (RUNX3), and the inflammatory mediator COX2; (e) excessive activation of PI3K/Akt, STAT3, and NF-кB pathways fundamental to cell proliferation and survival; (f) germline mutations in the Fanconi anemia/breast cancer susceptibility gene (Fanc/Brca) pathway, which coordi- 


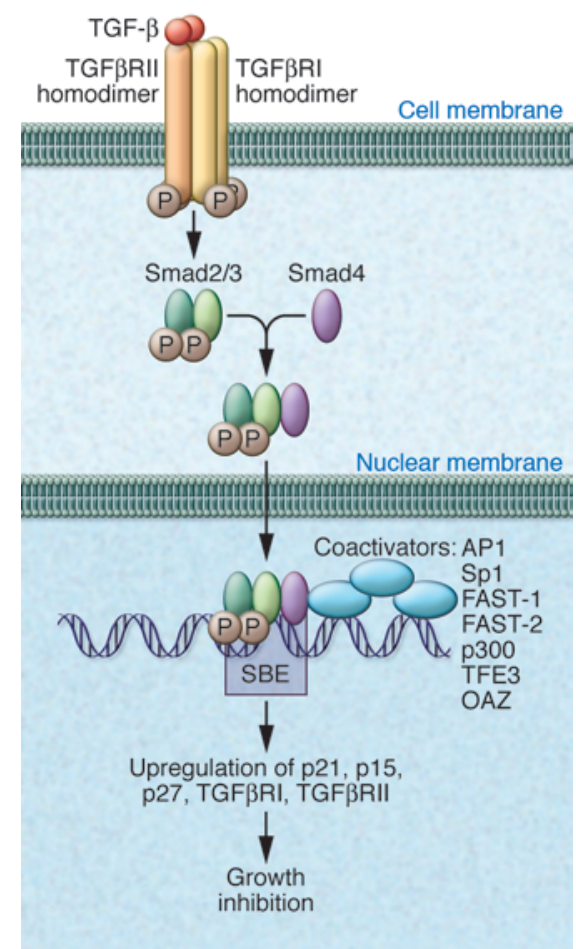

Figure 1

TGF- $\beta$ signaling. A TGF- $\beta$ dimer is shown binding to a homodimer of TGF $\beta$ RII, which then recruits a TGF $\beta R$ I homodimer. The heterotetrameric complex allows TGF $\beta R$ I to undergo serine-threonine phosphorylation, which activates its kinase activity and phosphorylates Smad2 and Smad3. These phosphorylated Smad proteins associate with Smad4, and the resultant complex translocates to the nucleus, where it can interact with coactivators such as AP1, Sp1, FAST-1 and the related transcription factor FAST-2, p300, TFE3, and OAZ (8-10) and corepressors (not shown) to modulate gene transcription. For example, genes encoding p21, p15, and p27 are upregulated through interactions between Smad2/3/4-coactivator complexes and Smad-binding elements (SBE). The upregulated proteins then act to inhibit cell-cycle progression and proliferation.

nates DNA cross-link repair; (g) susceptibility to loss of heterozygosity ( $\mathrm{LOH}$; the loss of normal function of one allele of a gene when the other allele has already been inactivated); and (h) multiple alterations in TGF- $\beta$ signaling pathways (2-6).

\section{Overview of TGF- $\beta$ signaling}

To better understand the study reported by Bornstein and colleagues in this issue of the JCI (7), it is important to review the signaling pathways that are activated by TGF- $\beta \mathrm{s}$; these are encoded by genes that belong to the TGF- $\beta$ superfamily, which includes three mammalian TGF- $\beta$ isoforms, activ- ins, inhibins, myostatin, growth and differentiation factors (GDFs), and bone morphogenetic proteins (BMPs). TGF- $\beta$ s regulate many cellular processes; enhance mesenchymal cell proliferation; inhibit epithelial cell proliferation; promote leukocyte infiltration; are chemotactic toward fibroblasts, monocytes, and blood polymorphonuclear cells; regulate the ECM; and support communications between the ECM and cellular cytoskeleton (8).

TGF- $\beta$ signaling is mediated by the type I and type II TGF- $\beta$ receptors (TGF $\beta$ RI and TGF $\beta$ RII, respectively) (Figure 1), which are transmembrane proteins with principally serine-threonine kinase activity (8-10). There are five different type II receptors that bind different members of the TGF- $\beta$ superfamily, occasionally binding to more than one member of the family. For example, there are two type II activin receptors, termed $\mathrm{A}$ and $\mathrm{B}$, that bind activin, nodal, and myostatin, and there is one type II BMP receptor that binds BMPs and GDFs (10). By contrast, there is only one TGF $\beta$ RII, and it only binds dimeric TGF- $\beta$ ligand (10). The ligand-bound TGF $\beta$ RII homodimer associates with a TGF $\beta$ RI homodimer, and the resultant heterotetrameric complex then initiates a signaling cascade (8-10) (Figure 1). TGF $\beta$ RI is phosphorylated within its GS region (i.e., SGSGSG sequence) by TGFßRII, allowing it to phosphorylate downstream receptor-regulated Smads (R-Smads) - Smad 2 and Smad3 - at their C-terminal SSXS motif $(10,11)$.

Once activated, R-Smads oligomerize with the common mediator Smad4, and the complex translocates to the nucleus, where, along with coactivators and corepressors, it regulates gene transcription (8-11) (Figure 1). Smad4 can then interact with protein complexes to modulate gene expression. Smad4, which lacks a C-terminal SSXS domain, associates (through a Smad-binding motif [CAGAC]) with many Smad-interacting DNA-binding proteins, including coactivators such as activator protein 1 (AP1), specificity protein 1 (Sp1), forkhead activin signal transducer 1 (FAST-1) and the related transcription factor FAST-2, p300, transcription factor for immunoglobulin heavy chain enhancer 3 (TFE3), and Olf-1/EBF-associated zinc finger (OAZ) (8-11). In contrast to the role of Smad4, Smad1, $-3,-5$, and 8 generally mediate the actions of BMPs, whereas inhibitory Smads (Smad6 and Smad7) act to suppress Smad2/3 activation (8-11).

\section{Deleterious role of TGF- $\beta$ pathways in cancer}

It is well established that components of the TGF- $\beta$ pathway act as tumor suppressors (11). Nonetheless, TGF- $\beta$ actions are context dependent, and increased TGF- $\beta$ expression has been observed frequently in the setting of perturbations in TGF- $\beta$ signaling pathways and in turn contributes to tumor growth in many epithelial cell-derived cancers $(10,11)$. TGF- $\beta$ 's tumor-promoting activity has been principally attributed to its paracrine effects: (a) enhancing tumor angiogenesis; (b) altering components of the ECM; (c) causing aberrant epithelial-mesenchymal interactions; (d) enhancing substratum adhesiveness, which facilitates tumor metastasis; and (e) suppressing cancer-directed immune responses $(10,11)$.

There is also a strong correlation between malignant progression and loss of sensitivity of the cancer cells to the growth-inhibitory effects of TGF- $\beta$ s. This resistance to TGF- $\beta$-mediated growth inhibition may be due to a variety of alterations in TGF- $\beta$ signaling pathways: (a) decreased expression of TGF $\beta$ RII, as in gastric cancer, or TGF $\beta$ RI, as in prostate and pancreatic cancers (12-14); (b) mutations in the genes encoding both receptors, as in colon and gastric cancers $(15,16)$; (c) mutations in the Smad4 gene, as in pancreatic cancer (17); (d) Smad4 underexpression, as in HNSCC (7); or (e) overexpression of inhibitory Smad7, as in pancreatic cancer (18). Taken together, these observations support the concept that potent paracrine actions on tumor growth, which are exerted by high levels of TGF- $\beta$, are often associated with perturbations in TGF- $\beta$ tumor suppressor pathways.

\section{Smad4 haploinsufficiency and HNSCC}

In this issue of the JCI, Bornstein and colleagues (7) point out that loss of the Smad4 protein in patients with HNSCC is known to correlate with depth of invasion of the cancer cells, a more advanced pathologic stage, enhanced regional metastases, and decreased patient survival. Other alterations known to occur in the TGF- $\beta$ pathway in HNSCC include decreased expression or mutation of TGF $\beta$ RII, increased TGF- $\beta 1$ expression, and decreased levels of activated Smad2 $(6,19,20)$. However, (a) the relevance of these molecular alterations to Smad4associated oncogenesis in HNSCC is not well understood; (b) it is not known whether Smad4 loss contributes to HNSCC initia- 


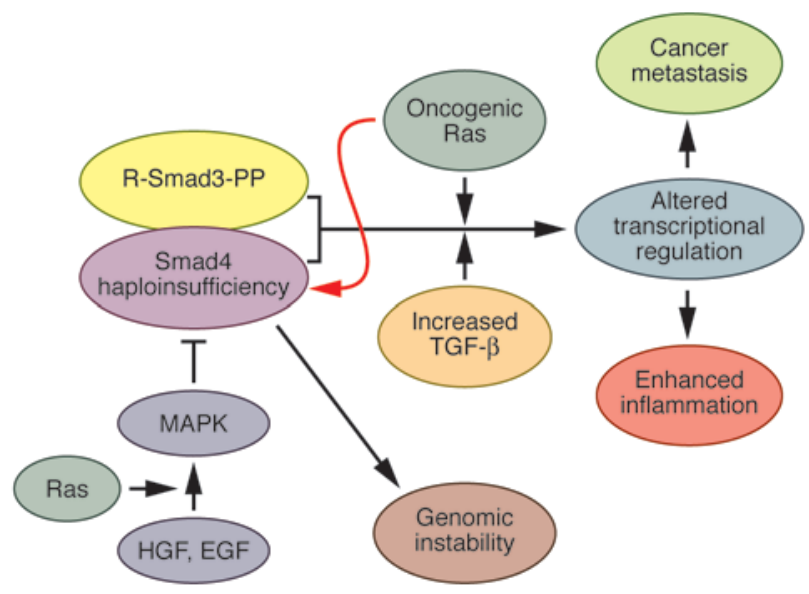

Figure 2

Consequences of Smad4 haploinsufficiency. As shown by Bornstein et al. in their study in this issue of the $\mathrm{JCl}(7)$, attenuated Smad4 levels in HNSCC lead to genomic instability, upregulation of phosphorylated R-Smad3 (R-Smad3-PP), excessive Ras activation (through overexpression or mutation), increased production of TGF- $\beta$ s, and enhanced inflammation. In theory, oncogenic Ras can enhance Smad4 degradation (red line), and ligands such as EGF or HGF can activate the Ras/MAKP pathway to further modulate Smad4 function. The net result is altered transcriptional regulation of numerous genes leading to enhanced cancer spread and metastasis.

tion and/or progression; and (c) previously studied animal models have not mimicked spontaneous human HNSCC. Accordingly, Bornstein et al. evaluated Smad4 expression in patients with HNSCC and studied a new mouse model of HNSCC.

Bornstein and colleagues (7) initially determined that the cancers in patients with HNSCC, as well as in the adjacent grossly normal buccal mucosa, expressed decreased levels of Smad4, which implied that Smad4 loss occurs early during the development of HNSCC in humans. The observation that Smad4 expression is also decreased in the normal buccal mucosa adjacent to head and neck cancer is an unexpected and exciting finding. In view of the fact that low levels of Smad4 have been associated with attenuated responsiveness to TGF- $\beta$-mediated growth inhibition, this observation suggests that Smad4 haploinsufficiency can lead to an enhanced propensity for head and neck carcinogenesis.

The mechanisms underlying decreased Smad4 expression in both HNSCC and the adjacent grossly normal mucosa are not readily evident (7) and cannot be explained by LOH alone. Additionally, it is not clear whether Smad4 levels within the tumor mass were decreased both in the cancer cells and in the stromal compartment, or only in the cancer cells. Nonetheless, in future studies it will be worth determining whether there is attenuation of Smad4 transcription rates or decreased Smad4
mRNA stability in HNSCC cancer cells and evaluating Smad4 expression in the different cell types within the tumor mass by in situ hybridization. Another exciting area of research would be to determine whether Smad4 mRNA levels are suppressed by noncoding microRNAs that are known to have the ability to target coding mRNAs. For example, the microRNA-23b cluster was recently reported to suppress Smad4 expression (21). next used an inducible head and neck-specific knockout system, which, after RU486 application to the oral cavity in 4-week-old mice, caused Smad4 deletion in head and neck epithelial cells (HN-Smad4-/- mice) (7). They observed the spontaneous development of oral tumors in $74 \%$ of $\mathrm{HN}$-Smad4 $4^{-/}$ mice, some of which developed lymph node metastases. By contrast, tumors did not form in either the HNSmad4 $4^{+-}$or the $\mathrm{HN}$-Smad $4^{+/+}$mice. These observations provide more direct evidence in support of the concept that Smad4 deficiency leads to an enhanced propensity for head and neck carcinogenesis.

The ability of Smad4 deficiency to cause HNSCC (7) was somewhat surprising, given the previous observation that in a mouse model of HNSCC in which TGF $\beta$ RII was deleted in head and neck mucosa, attenuation of growth inhibition did not lead to malignant transformation unless there was concomitant expression of mutated $K$-ras or
Importantly, Bornstein and colleagues
$H$-ras (6). The authors therefore postulated that the loss of Smad4 facilitated $K$-ras or $H$-ras activation (7). They sequenced both genes in 18 HNSCC tumors that have arisen in the $\mathrm{HN}$-Smad4 $4^{-/}$mice and determined that four tumors exhibited oncogenic $H$-ras mutations at codon 61 . Inasmuch as $H$-ras is more frequently mutated at codons 12 and 13, this observation suggests that Smad4 loss in head and neck epithelial cells in mice may specifically lead to codon $61 \mathrm{H}$-ras mutations, and this possibility is worthy of further exploration.

Bornstein and colleagues (7) also determined that tumors with wild-type Ras exhibited increased Ras protein levels, in agreement with the overall hypothesis that spontaneous Ras activation is a potential initiation event for HNSCC formation in $H N-S m a d 4^{-/-}$tumors. To test this hypothesis, the authors generated mice with heterozygous deletion of $S$ mad4 together with heterozygous $H N-K$-ras ${ }^{G 12 D}$ mutation in head and neck epithelia ( $\mathrm{HN}$-K-ras ${ }^{G 12 D} \mathrm{Smad} 4^{+/-}$mice) and observed that these mice developed HNSCC. By contrast, HN-Smad4 ${ }^{+/-}$mice did not develop tumors. Therefore, in this mouse model, Smad4 haploinsufficiency did not lead to cancer formation, except in the presence of oncogenic $K$-ras, underscoring differences between the mouse model and HNSCC in humans.

\section{Smad4 and genomic stability}

To explain their findings with respect to the high incidence of Ras mutation or overexpression in the inducible head and neck specific-knockout system (HN-Smad4-/- mice), Bornstein and colleagues (7) postulated that Smad4 deletion in head and neck epithelium resulted in genomic instability. Indeed, they observed decreased expression of mRNA moieties encoding members of the Fanconi anemia (FA) family of proteins and Rad51, which are known to participate in the DNA repair pathway by regulating homologous recombination (22). Thus, there were variable but significant decreases in the levels of mRNA encoding Brca1, FancA, FancD2, and Rad51 in both the HNSCC lesions and adjoining mucosa of $\mathrm{HN}$-Smad4 $4^{-/}$mice, indicating that these changes most likely occurred as a result of Smad4 deletion and prior to tumor formation.

Two additional lines of evidence supported the concept that Smad4 deficiency leads to genomic instability in HNSCC. First, there was an increase in centrosome number in the HNSCC lesions of the HN-Smad4 $4^{-1-}$ mice (7). Second, as determined by array 
comparative genomic hybridization, the tumors in the HN-Smad4 ${ }^{-/-}$mice exhibited chromosomal losses that are syntenic to human chromosomal regions $19 \mathrm{p} 13$ and $17 \mathrm{q} 21$ and are common in human HNSCC (4). Taken together, these findings indicate that Smad4 has a gatekeeper function, maintaining genomic stability in oral mucosa.

TGF- $\beta 1$ can cooperate with Ras proteins to promote epithelial-mesenchymal transformation (EMT), a process whereby epithelial cells acquire mesenchymal markers; cell-cell junctional integrity is lost; the epithelial adhesion molecules $\beta$-catenin and E-cadherin become mislocalized principally to the nucleus and cytoplasm, respectively; and cellular invasiveness is enhanced (11). Inasmuch as one of the consequences of Smad4 deletion in $\mathrm{HN}$-Smad4 $4^{-/-}$mice was increased expression of TGF- $\beta 1$, it is likely that the initiating event occurring as a consequence of excessive Ras activation was further fueled by elevated TGF- $\beta 1$ levels.

\section{HNSCC and inflammation}

Excessive Ras activation may also redirect TGF- $\beta$ actions toward tumor promotion and a proinflammatory profile (11). In this regard, Bornstein and colleagues (7) show that the stroma adjacent to both the normal buccal mucosa and to the HNSCC in the HN-Smad4 $4^{-/-}$mice exhibited leukocyte infiltration comprising in part macrophages, granulocytes, and $\mathrm{T}$ lymphocytes (including Th17 cells, which represent a subset of proinflammatory $\mathrm{T}$ lymphocytes that are activated by TGF- $\beta 1$ in mice). Moreover, there was increased cytokine expression, including elevated levels of monocyte chemoattractant protein-1 (MCP-1), MCP-2, and macrophage inflammatory protein-2 (MIP-2), which are known to be upregulated by TGF- $\beta 1$ overexpression in keratinocytes (23). These alterations occurred in spite of the loss of nuclear phospho-Smad2 (pSmad2), which points to deficient Smad2-dependent signaling. However, this defect was counterbalanced by increased nuclear staining for Smad3, implying selective aberrant activation of Smad3-dependent pathways. To test for this possibility, $\mathrm{HN}-\mathrm{Smad} 4^{-/-}$mice were crossed into a $\mathrm{Smad3}^{+/-}$background, yielding $\mathrm{HN}$-Smad4 $4^{-/-} \mathrm{Smad}^{+/-}$mice (7). The lesions in these mice exhibited reduced leukocyte infiltration in comparison with the $\mathrm{HN}$-Smad4 $4^{--}$animals, implicating TGF- $\beta$ activated Smad3 pathways in the cancerassociated inflammatory process.
Surprisingly, there was also increased staining for pSmad1/5/8 (7). It is not clear, however, whether activation of these Smads was due to excessive activation of BMPdependent pathways, since the expression of BMP and/or BMP receptors was not examined, or to TGF- $\beta$ acting through an alternate $\mathrm{pSmad} 1 / 5$ pathway, as recently reported in certain cell types, including epithelial cells (24).

\section{Possible implications}

The present findings in HNSCC (7), which were largely focused in a mouse model of human HNSCC, underscore the potential importance of Smad4 as a "guardian gene" and raise the possibility that cancers in which there is concomitant loss of Smad4 and excessive ras oncogene activation may become especially susceptible to marked genomic instability. For example, Smad4 was originally identified as a mutated/ deleted gene in pancreatic ductal adenocarcinoma (PDAC), and its original name was deleted in pancreatic cancer, locus 4 (19). PDAC is also characterized by a greater than $90 \%$ mutation rate in the $K$-ras oncogene (25). The concomitant loss of Smad4, observed in $50 \%$ of PDAC cases, and high frequency of oncogenic $K$-ras in this cancer could explain the marked genomic instability observed in the malignancy. In view of the known ability of oncogenic Ras to induce Smad4 degradation (26), and of tyrosine kinase pathways that activate Ras to modulate Smad4 function (27), it is also possible that once a mucosal cell exhibits low levels of Smad4 and its Ras pathways become excessively active, a vicious cycle is established that leads to a further lowering of Smad4 levels as a consequence of Rasinduced Smad4 degradation (Figure 2). In conjunction with the ensuing genomic instability, which elicits multiple molecular alterations including a pro-oncogenic inflammatory profile, these alterations can then promote disease aggressiveness, invasion, and metastasis.

\section{Future directions}

It is not known why Smad4 levels are low in both HNSCC and the adjacent grossly normal mucosa (7). Clearly, future studies are necessary to examine Smad4 transcriptional regulation in head and neck mucosa, the potential role of microRNAs in this regulation, the role of endogenous inhibitors of transcription such as noncoding antisense RNA, and the role of translational modifications such as ubiquitina- tion and sumoylation (28). Similarly, it is not clear exactly how a full dose of Smad4 acts to maintain genomic stability. Differential gene expression studies in relation to different levels of Smad4 may shed light on this issue.

$K$-ras and $H$-ras mutations are more common in Asian populations with HNSCC, whereas in the United States, HNSCC is more frequently associated with elevated $K$-ras and $H$-ras levels (6). In view of the important ras-Smad4 interactions, additional studies should be conducted to determine whether these regional differences are due to specific dietary, environmental, or genetic factors. Similarly, in view of the inflammatory response observed as a result of ras-Smad4 interactions, and the activation of Smad1/5 pathways, it will be important to determine whether other noncanonical TGF- $\beta$ signaling molecules, such as JNK and p38 MAPK kinase, are activated in HNSCC and whether reactive oxygen species or specific cytokines contribute to the inflammatory response.

Future studies may take advantage of the inducible head and neck-specific Smad4knockout mouse model (7) to evaluate chemoprevention strategies and to test a variety of therapeutic mechanisms that concomitantly target Ras, Smad4, and TGF- $\beta$ signaling pathways, in an attempt to suppress genomic instability, aberrant proliferation, and lymph node metastasis.

\section{Acknowledgments}

This work was supported in part by Public Health Service Grant R37-CA-75059 awarded by the NIH to M. Korc.

Address correspondence to: Murray Korc, Department of Medicine, DartmouthHitchcock Medical Center, 1 Medical Center Drive, Lebanon, New Hampshire 03755, USA. Phone: (603) 650-7936; Fax: (603) 650-6122; E-mail: murray.korc@ dartmouth.edu.

1. Forastiere, A., Koch, W., Trotti, A., and Sidransky, D. 2001. Head and neck cancer. N. Engl. J. Med. 345:1890-1900.

2. Hunter, K.D., Parkinson, E.K., and Harrison, P.R. 2005. Profiling early head and neck cancer. Nat. Rev. Cancer. 5:127-135.

3. Lu, S.L., Herrington, H., and Wang, X.J. 2006. Mouse models for human head and neck squamous cell carcinomas. Head Neck. 28:945-954.

4. Weber, F., et al. 2007. Microenvironmental genomic alterations and clinicopathological behavior in head and neck squamous cell carcinoma. JAMA. 297:187-195.

5. Marsit, C.J., et al. 2004. Inactivation of the Fanconi anemia/BRCA pathway in lung and oral cancers: implications for treatment and survival. Oncogene. 
23:1000-1004.

6. Lu, S.L., et al. 2006. Loss of transforming growth factor-beta type II receptor promotes metastatic head-and-neck squamous cell carcinoma. Genes Dev. 20:1331-1342.

7. Bornstein, S., et al. 2009. Smad4 loss in mice causes spontaneous head and neck cancer with increased genomic instability and inflammation. J. Clin. Invest. 119:3408-3419.

8. Pardali, E., and ten Dijke, P. 2009. Transforming growth factor-beta signaling and tumor angiogenesis. Front. Biosci. 14:4848-4861.

9. Kang, J.S., Liu, C., and Derynck, R. 2009. New regulatory mechanisms of TGF-beta receptor function. Trends Cell Biol. 19:385-394.

10. Massagué, J. 2008. A very private TGF-beta receptor embrace. Mol. Cel. 29:149-150

11. Massagué, J. 2008. TGF beta in cancer. Cell. 134:215-230.

12. Park, K., et al. 1994. Genetic changes in the transforming growth factor $\beta$ (TGF- $\beta$ ) type II receptor gene in human gastric cancer cells: Correlation with sensitivity to growth inhibition by TGF- $\beta$. Proc. Natl. Acad. Sci. U. S. A. 91:8772-8776.

13. Kim, I.Y., et al. 1996. Genetic change in transforming growth factor beta (TGF-beta) receptor type I gene correlates with insensitivity to TGF-beta 1 in human prostate cancer cells. Cancer Res. 56:44-48.
14. Baldwin, R.L., et al. 1996. Attenuated ALK5 receptor expression in human pancreatic cancer: Correlation with resistance to growth inhibition. Int. J. Cancer. 67:283-288.

15. Markowitz, S., et al. 1995 . Inactivation of the TGF- $\beta$ type II receptor in colon cancer cells with microsatellite instability. Science. 268:1336-1338.

16. Myeroff, L.L., et al. 1995. A transforming growth factor beta receptor type II gene mutation common in colon and gastric but rare in endometrial cancers with microsatellite instability. Cancer Res. 55:5545-5547.

17. Hahn, S.A., et al. 1996. DPC4, a candidate tumor suppressor gene at human chromosome 18q21.1. Science. 271:350-353.

18. Kleeff, J., et al. 1999. The TGF-beta signaling inhibitor Smad7 enhances tumorigenicity in pancreatic cancer. Oncogene. 18:5363-5372.

19. Muro-Cacho, C.A., Rosario-Ortiz, K., Livingston, S. and Muñoz-Antonia, T. 2001. Defective transforming growth factor beta signaling pathway in head and neck squamous cell carcinoma as evidenced by the lack of expression of activated Smad2. Clin. Cancer Res. 7:1618-1626.

20. Lu, S.L., et al. 2004. Overexpression of transforming growth factor beta 1 in head and neck epithelia results in inflammation, angiogenesis, and epithelial hyperproliferation. Cancer Res. 64:4405-4410.

21. Rogler, C.E., et al. 2009. MicroRNA-23b cluster
microRNAs regulate transforming growth factorbeta/bone morphogenetic protein signaling and liver stem cell differentiation by targeting Smads. Hepatology. 50:575-584.

22. Kennedy, R.D., and D'Andrea, A.D. 2005. The Fanconi anemia/BRCA pathway: new faces in the crowd. Genes Dev. 19:2925-2940.

23. Li, A.G., Wang, D., Feng, X.H., and Wang, X.J. 2004. Latent TGFbeta1 overexpression in keratinocytes results in a severe psoriasis-like skin disorder. EMBO J. 23:1770-1781.

24. Liu, I.M., et al. 2009. TGF beta-stimulated Smad1/5 phosphorylation requires the ALK5 L45 loop and mediates the pro-migratory TGF beta switch. EMBO J. 28:88-98.

25. Korc, M. 1998. Role of growth factors in pancreatic cancer. Surg. Oncol. Clin. N. Am. 7:25-41.

26. Saha, D., Datta, P.K., and Beauchamp, R.D. 2001 Oncogenic ras represses transforming growth factor-beta/Smad signaling by degrading tumor suppressor Smad4. J. Biol. Chem. 276:29531-29537.

27. Kretzschmar, M., Doody, J., Timokhina, I., and Massagué, J. 1999. A mechanism of repression of TGFbeta/Smad signaling by oncogenic Ras. Genes Dev. 13:804-816.

28. Long, J., Wang, G., He, D., and Liu, F. 2004. Repression of Smad4 transcriptional activity by SUMO modification. Biochem. J. 379:23-29. 